\title{
Eddy Current Effect of the BNL-AGS Vacuum Chamber on the Optics of the BNL-AGS Synchrotron*
}

\author{
N. Tsoupas*, L. Ahrens, K.A. Brown, J. W. Glenn, K. Gardner \\ Brookhaven National Laboratory, Upton, NY 11973 USA
}

\begin{abstract}
During the acceleration cycle of the AGS synchrotron, eddy currents are generated within the walls of the vacuum chambers of the AGS main magnets. The vacuum chambers have elliptical cross section, are made of inconel material with a wall thickness of $2 \mathrm{~mm}$ and are placed within the gap of the combined-function main magnets of the AGS synchrotron. The generation of the eddy currents in the walls of the vacuum chambers, creates various magnetic multipoles, which affect the optics of the AGS machine. In this report these magnetic multipoles are calculated for various time interval starting at the acceleration cycle, where the magnetic field of the main magnet is $\sim 0.1 \mathrm{~T}$, and ending before the beam extraction process, where the magnetic field of the main magnet is almost constant at $\sim 1.1 \mathrm{~T}$. The calculations show that the magnetic multipoles generated by the eddycurrents affect the optics of the AGS synchrotron during the acceleration cycle and in particular at low magnetic fields of the main magnet. Their effect is too weak to affect the optics of the AGS machine during beam extraction at the nominal energies.
\end{abstract}

\section{INTRODUCTION}

The AGS synchrotron has twelve super-periods with 20 combined-function magnets[1] per super-period. Every two consecutive combined function magnets are paired to form either a horizontally focusing or defocusing quadrupole. The cross section of a horizontally focusing combined-function magnets used in the AGS synchrotron is shown in Figures 1a(C-type), and Figure 1b(B-type). The horizontally defocusing magnets are made of the same magnets ( $B$, and $C$ type) but with the magnets rotated $180^{\circ}$ about the vertical axis. The cross section of the elliptical in shape vacuum chamber is also shown in the figure 1a.

Simulations of the beam optics of the AGS synchrotron are normally done using the MAD computer code[2], the BEAM code[3] or a modified version of the RAYTRACE code[4]. In these simulations the quadrupole strength $(\mathrm{K} 1)$ and sextupole strength $(\mathrm{K} 2)$ of the combined function magnets is used, and their values are obtained from static magnetic field calculations [5] or from the experimental magnetic field maps of the AGS

\footnotetext{
* Work supported by the U.S. Department of Energy

\$ email tsoupas@bal.gov
}

magnets[6]. In either case, both the quadrupole and sextupole strengths of the combined function magnets were obtained from static calculations, and did not include any contributions from eddy-currents generated in the vacuum chamber during the acceleration cycle. Measurements on the chromaticity of the AGS synchrotron have shown[7,8,9,10,11] that the chromaticity of the AGS synchrotron is affected by the ramp rate of the AGS main magnets. These measurements were used to generate a momentum dependent magnetic sextupole strength to be used as input data in the MAD computer code for beam optics simulations of the AGS synchrotron.

It is the purpose of this report to calculate the magnetic sextupole produced by the eddy-currents formed in the AGS vacuum chamber, during the ramping of the main magnets. Subsequently to use these magnetic sextupole strengths to calculated the chromaticy of the AGS synchrotron and make comparison with experimental measurments.

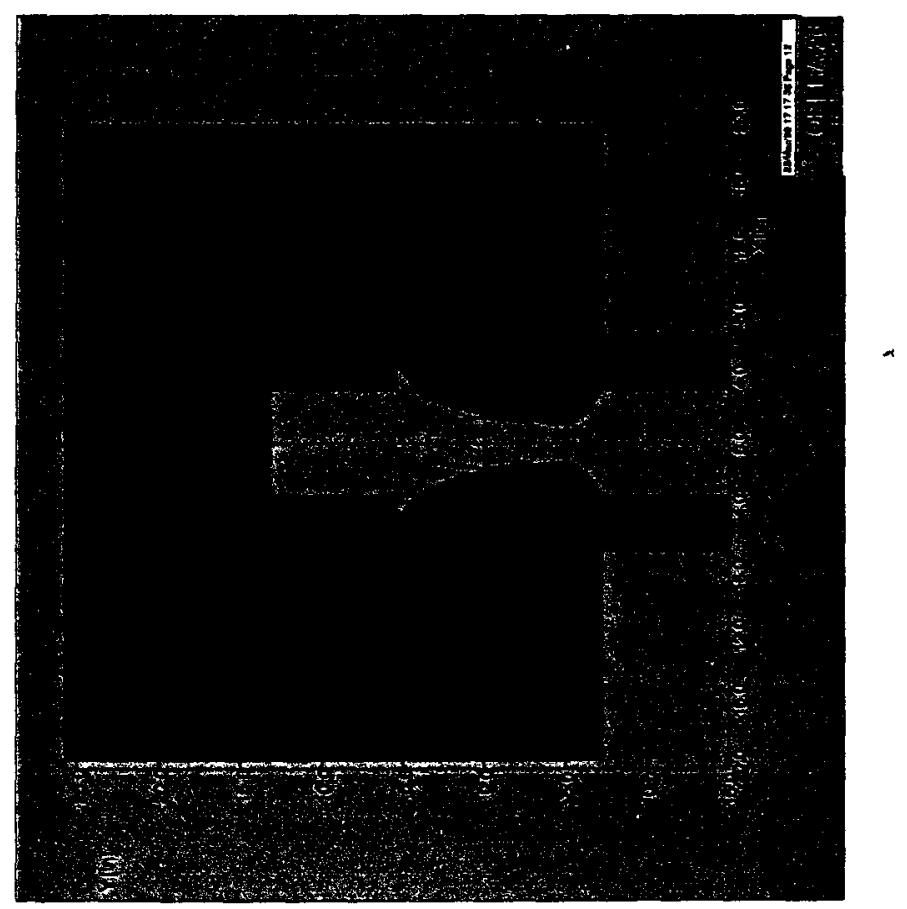

Figure 1a. Cross section of a C-type combined function AGS main magnet. The vacuum chamber is shown between the gap of the magnet. The magnet shown in this orientation is horizontally focusing. By rotating the magnet $180^{\circ}$ about the $y$-axis which passes through the vacuum chamber, the magnet becomes horizontally defocusing. 


\section{MAGNETIC SEXTUPOLE OF THE AGS MAIN MAGNETS}

The magnetic sextupole component of the AGS main magnetic field will affect the chromaticity $\xi$ of the synchrotron according to the formulae:

$$
\begin{aligned}
& \xi_{x} \propto-\frac{1}{Q_{x}} \oint \beta_{x}(s) b_{2}(s) \eta_{x}(s) d s \\
& \xi_{y} \propto-\frac{1}{Q_{y}} \oint \beta_{y}(s) b_{2}(s) \eta_{x}(s) d s
\end{aligned}
$$

Where $\beta_{x}(s)$ and $\beta_{y}(s)$ are the horizontal and vertical beta functions, $Q_{x}$ and $Q_{y}$ are the betatron tunes, $\eta_{x}(s)$ is the dispersion function, and $b_{2}(s)$ is a quantity proportional to the sextupole strength, along the synchrotron.

The quantity $b 2$ which is the sextupole component of the magnetic field generated by the $\Lambda \mathrm{GS}$ main magnet, has been calculated as a function of the $\mathrm{B}_{0}$ dipole field of the main AGS main magnet in two ways:

a) Static 2-dimentional calculations

b) Transient 2-dimentional calculations

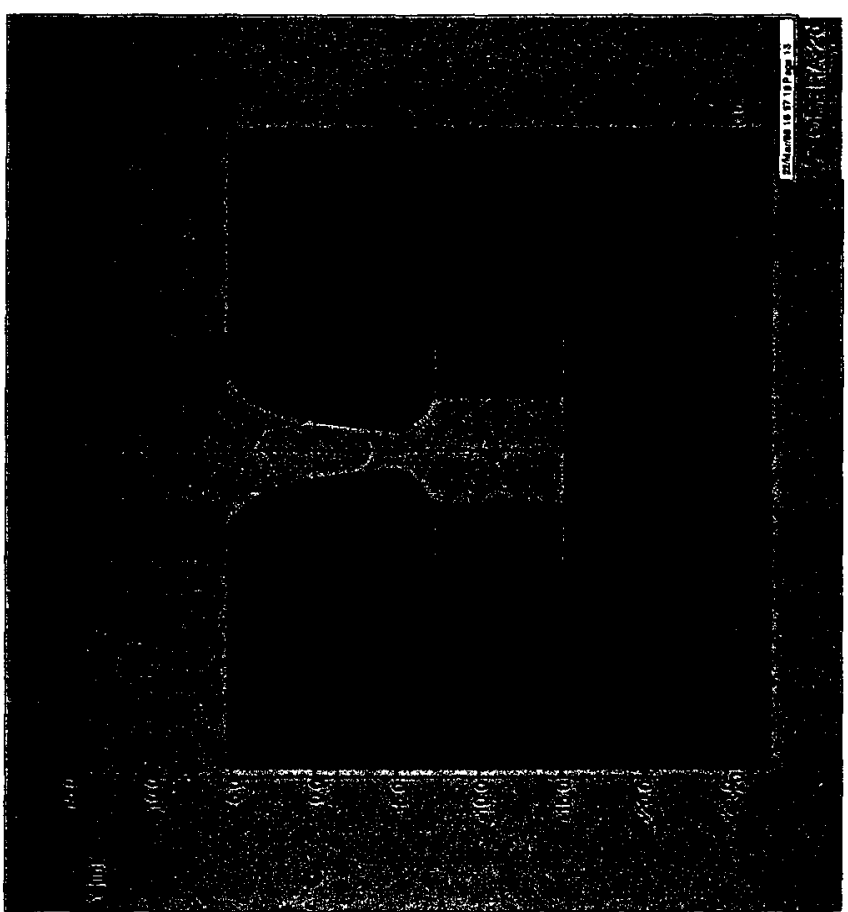

Figure $1 b$. Cross section of a B-type AGS main magnet. The magnet shown in this orientation is horizontally focusing. By rotating the magnet $180^{\circ}$ about the y-axis, passing through the vacuum chamber, the magnet becomes horizontally defocusing.

\subsection{Static Calculations}

The static calculations were performed using a 2-D computer code[12] for electroinagnetic calculations. The C and B type AGS main magnets were modeled based on the cross sections shown in Figures $1 \mathrm{a}$ and $1 \mathrm{~b}$. In the calculations, the default nonlinear material, which is provided by the code, was used for the permiability of the iron. The quantity $b_{2}$ (s) appearing in the equations $1 a$ and $1 \mathrm{~b}$ is the coefficient of the quadratic term in the expansion (as function of $x$ ) of the vertical component $\left(B_{y}\right)$ of the magnetic field at the median plane of the magnet.

$B_{y}(x)=B_{0}\left(1+b_{1} x+b_{2} x^{2}+b_{3} x^{3}+\ldots.\right)$

In equation (2) the variable $x$ is the distance from the longitudinal axis of the magnet, on the horizontal plane. Figure 2 (dashed line) shows a plot of the quantity K2 = $2 b_{2} /(B \rho)$ (as defined in the computer code MAD[2]) as a function of the excitation field $B_{y}(x=0, y=0)$. The calculated values of the sextupole strength $K 2$ for the $C$ type magnet (see Fig.1a) are almost the same ${ }^{1}$ with those of the B-type magnet ${ }^{2}$. This is due to the fact that both type of magnets have the same geometrical shape of the pole tip therefore the magnetic field within the gap is iron dominated at these excitation currents.

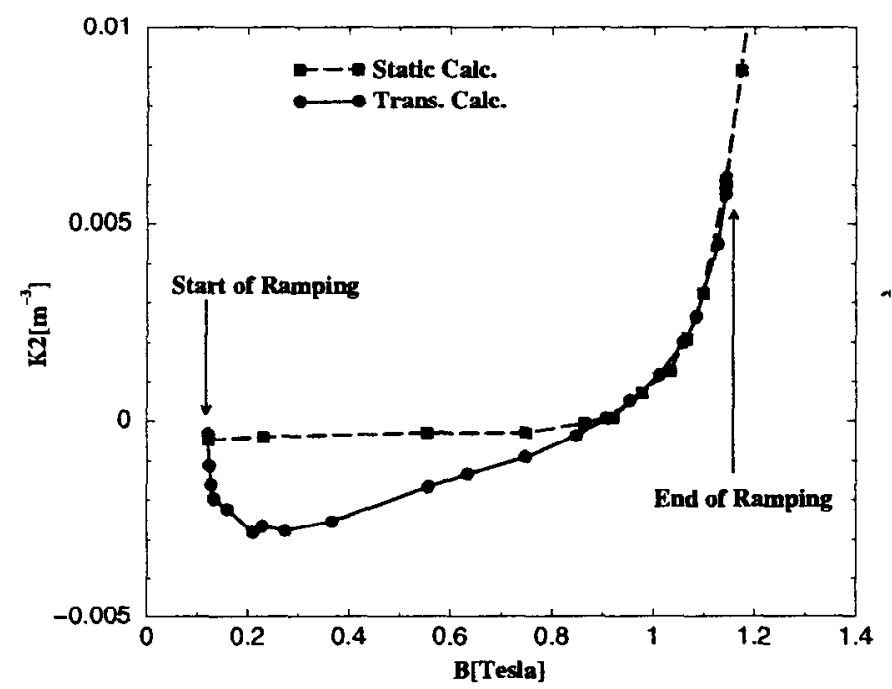

Figure 2: The magnetic sextupole strength $K 2$ of the main magnetic field as a function of the $B_{y}(x=0, y=0)$ (main magnetic field). The dashed line which connects the squares, corresponds to the $\mathrm{K} 2$ values from the static case. The solid line connecting the circles, corresponds to the calculations of $\mathrm{K} 2$, obtained from the transient case.

\footnotetext{
${ }^{1}$ The difference is $-1 \%$. This value is within the error of the calculations.

${ }^{2}$ The sextupole strength $K 2$ remains the same for both focusing and defocusing magnets.
} 


\subsection{Transient Culculations}

The transient magnetic field calculations were performed using the same computer code as in the static calculations, but the transient 2-D version. The excitation current $I(t)$ is required as input data in the transient calculations. The function $\mathrm{I}(\mathrm{t}) / \mathrm{m} \max$ is plotted in Figure 3 as a function of time. In the same Figure 3 the derivative $\mathrm{dI}(\mathrm{t}) / \mathrm{dt}$ is also plotted. The input data allow the generation of eddy currents in the coils of the magnet which are made of copper, and the wall of the vacuum chamber which is made of inconel. The magnet iron was assumed to have zero conductivity. This was a reasonable assumption since the iron core is made of electrically isolated laminations $0.031^{\prime \prime}$ thick. These laminations are held together by two iron plates 1" thick, each placed at

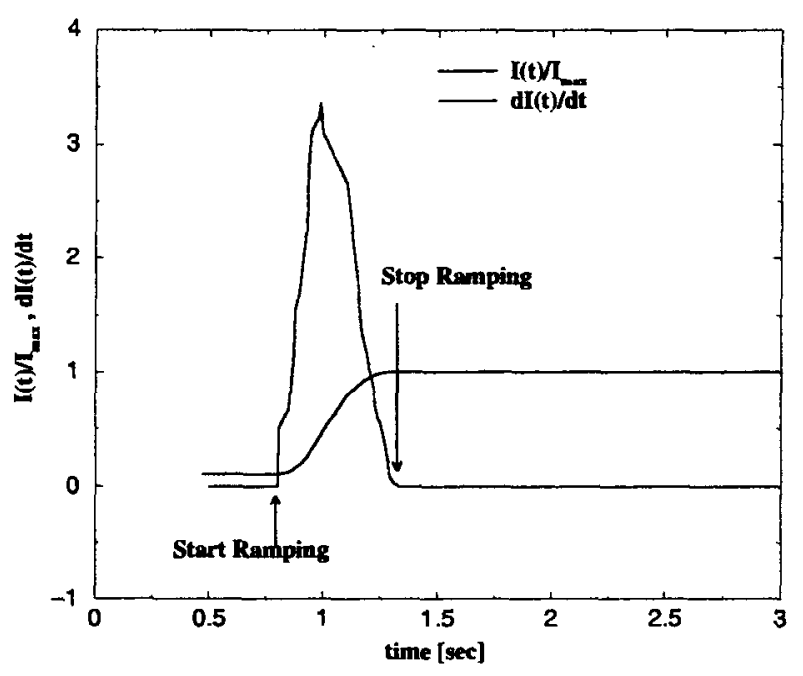

Figure 3: Excitation current of the main AGS magnets as a function of time (dased curve). The solid curve is the dI $(t) / d t$ curve.

the end of the magnets to compress the laminations. As in the static case, the sextupole strength $\mathrm{K} 2$ of the magnetic field was calculated as a function of the magnet excitation and is plotted in Figure 2. A comparison of the sextupole strength $\mathrm{K} 2$ between the static and transient cases, shows that the eddy currents of the vacuum chamber affect the sextupole component of the main magnetic field but only at low excitation of the magnet. At higher excitations of the magnet the $\mathrm{K} 2$ values for both static and transient, coincide even before the end of the ramping (see Fig. 2).

The calculated quadrupole strength $\mathrm{K} 1$ of the magnetic field yields the same values for both, the static and the transient case, at any excitation level of the magnet.

\section{FURTHER STUDIES}

The 2-D calculations discussed above provide enough information to calculate the chromaticity of the AGS synchrotron during the acceleration cycle. The chromaticity chalculations can be done by using the MAD computer code. A more rigorous study calls for a 3-D transient calculations which can take into account the eddy-currents induced in each of the 1" thick plates which are placed at each end of the magnet to hold the laminations together. These 3-D calculations will be performed, in the near future as an ongoing effort of this task, using the computer code[12] which computes magnetic fields in 3-dimensions. Such calculations will provide a more accurate value of the sextupole strength $\mathrm{K} 2$, which can be used to calculate the chromaticity of the AGS synchrotron.

Experimental measurements of the chromaticity of the AGS synchrotron will be performed as a function of the magnetic field of the main magnet while the main magnet is ramping up, at various rates $\mathrm{dI}(\mathrm{t}) / \mathrm{dt}$.

\section{REFERANCES}

[1] E. B leser "The Optimum Central Orbit in the AGS" ADT Note No. 217

E. Bleser "Where are the AGS Magnets" ADT Note No. 215

[2] Hans Grote, and F.Christoph Iselin "The MAD Program" CERN/SL/90-13(AP)

[3] N. Tsoupas et. al. "Closed Orbit Calculations at AGS and Extraction Beam Parameters at H13" BNL. AD/RHIC/RD-75

[4] N. Tsoupas et. al. "Stable Spin Direction of a Polarized Proton Beam at the Injection Poin of RHIC" BNL AGS/RHIC/SN No. 021

[5] W. Meng "Three Dimensional Field Analysis for the AGS Combined Function Magnets" PAC93 May 17-20 1993

[6] R. Thern "experimental Magnetic Field Maps of the AGS magnets" (private comunication)

[7] E. Auerbach et. al. "Comments on the AGS Chromaticity" BNL AGS/AD/Tech. Note No. 276

[8] E. Bleser "Comments on the AGS Chromaticity: II" BNL AGS/AD/Tech. Note No. 288

[9] K. Gardner and L. Ahrens BNL 36490, 1985 Particle Accelerator Conference, $5 / 13 / 85$

[10] L. Ahrens, and W. K. van Asselt AGS SR-206, 6/26/86.

[11] K. Gardner et. al. AGS SR-226, 6/7/87.

[12] Vector Fields Inc. 